\title{
Measurement of cross sections in Higgs boson decays to two photons with the ATLAS detector
}

\author{
loannis Nomidis* on behalf of the ATLAS Collaboration \\ LPNHE-Paris/CNRS, France \\ E-mail: ioannis.nomidisecern.ch
}

\begin{abstract}
Higgs boson decays to two photons can be selected with high efficiency, and the very good invariant mass resolution allows a robust subtraction of the backgrounds. These proceedings present measurements of differential cross sections, as well as cross section measurements for the different Higgs boson production processes in the simplified template cross section framework using $p p$ collision data collected at $13 \mathrm{TeV}$ during Run-2 operations of the LHC with the ATLAS detector. The differential cross sections are interpreted in the context of an Effective Lagrangian to set constraints on possible new interactions of the Higgs boson with the Standard Model particles. Moreover, the differential measurement of the transverse momentum of the Higgs boson is used to set limits on a possible modification of the Yukawa coupling of the Higgs boson to the charm quark.
\end{abstract}

European Physical Society Conference on High Energy Physics - EPS-HEP2019 -

10-17 July, 2019

Ghent, Belgium

\footnotetext{
* Speaker.
} 


\section{Introduction}

During Run-2 $p p$ operations of the LHC at $13 \mathrm{TeV}$, an increasingly coherent picture of the properties of the Higgs boson has been drawn thanks to a wide range of measurements, particularly by establishing its coupling to the third generation of quarks with the observation of the $H \rightarrow$ $b \bar{b}$ decay and $t t H$ production mode. The $H \rightarrow \gamma \gamma$ decay which, together with $H \rightarrow Z Z^{*} \rightarrow 4 \ell$, led to the discovery of the Higgs boson, played a significant role in the $t t H$ observation as well as in measurements of differential cross sections with the first Run-2 data from the 2015-2016 operations. Preliminary measurements have shown no significant deviations from the Standard Model (SM) predictions. Therefore the goal for analyses of the full Run-2 data is to keep systematic uncertainties under control, despite the increased pile-up in the 2017-2018 data, in order to achieve the best possible precision and probe smaller deviations from the SM. ATLAS [1] maintained excellent photon performance throughout Run-2 allowing the analysis of the diphoton final state to yield new results with $80-139 \mathrm{fb}^{-1}$.

Various complementary approaches are being pursued for the study of the Higgs boson properties. Analyses targeting direct measurements of the cross-section modification or coupling modification provide more direct tests of the SM but inevitably use many assumptions. On the other hand, measurements of fiducial cross sections of the Higgs boson, inclusive in production mode, require far less assumptions and are largely model independent. The Simplified Template Cross Sections (STXS) framework [2,3] is the middle ground between these two approaches in order to perform finely-grained measurements of individual production modes in various kinematic regions with minimal theory assumptions. Its design aims to exploit the power of combining multiple decay channels which have different sensitivities in different parts of the kinematic phase space. Eventually, both fiducial differential cross sections and STXS measurements can be used in various interpretations in order to set constraints on new physics.

In these proceedings, the ATLAS results from the analysis of the 2015-2017 dataset $\left(80 \mathrm{fb}^{-1}\right)$ for the cross sections of the various production modes of the Higgs boson with the STXS approach are discussed shortly. Then the new fiducial cross-section measurements with the full Run-2 dataset $\left(139 \mathrm{fb}^{-1}\right)$ are presented, followed by interpretations of the differential cross sections in the context of an Effective Field Theory (EFT) and Yukawa coupling of the Higgs boson to the charm quark.

\section{Cross-section measurements per production mode}

The latest STXS measurements are available from the analysis of $80 \mathrm{fb}^{-1}$ of data [4]. They are performed for a reduced version of stage-1, in bins defined by fiducial selections that target the different production modes and are split in kinematics of the Higgs boson and jets associated with its production. The separation of the production modes at reconstruction level is following closely the fiducial selections and, when available statistics allows, further splitting is used in order to exploit regions of the phase space with high detector resolution and/or low expected backgrounds. The sensitivity of the measurements is further improved with the use of boosted decision trees in order to separate signal from different production modes and background. In each bin, the crosssection times branching ratio is measured and Figure 1a shows the corresponding ratio of each measurement to the SM prediction. All measurements are in very good agreement with the SM 
predictions within $1 \sigma$. It is worth noting, though, that they are statistically limited and therefore such measurements will improve with the analysis of the full Run-2 data.

In the above set of measurements, that of the $t t H$ production mode has been repeated using the full Run-2 dataset of $139 \mathrm{fb}^{-1}$ and improvements in the photon identification and jet energy calibration [5]. Benefiting mainly from the larger dataset, $t t H$ production is observed with $4.9 \sigma$ significance, as demonstrated by the excess in the diphoton invariant mass of events falling in $t t H$ enriched categories (Figure 1b). The observation corresponds to a signal strength measurement of $\mu_{t t H}=1.38_{-0.36}^{+0.41}$. With this result, we have a single-channel observation almost at the $5 \sigma$ level of the $t t H$ production mode serving as strong direct evidence of Higgs boson couplings to the top quark.

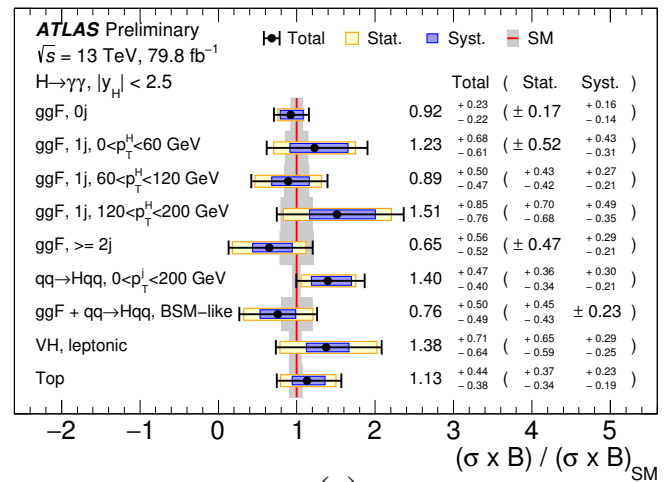

(a)

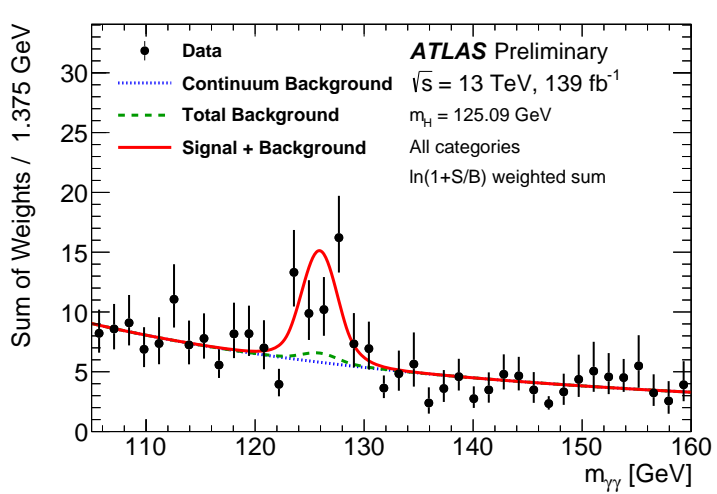

(b)

Figure 1: (a) Ratios of measured cross-sections times diphoton branching ratio to their SM predictions for each production mode, as defined in a reduced version of STXS stage-1, performed with $80 \mathrm{fb}^{-1}$ [4]. (b) The weighted diphoton invariant mass spectrum obtained from the sum of multiple analysis categories targeting $t t H$ production with the full Run-2 dataset of $139 \mathrm{fb}^{-1}$ [5].

\section{Fiducial differential cross sections}

New measurements of differential and total fiducial cross sections have been performed with the full Run-2 data [6]. For model-independent measurements, we define a fiducial region directly accessible experimentally with the ATLAS detector and make no attempt to separate production modes. Acceptance corrections for the extrapolation of the measurement from the fiducial to the full phase space are largely model dependent, as they rely on assumptions (i.e. physics model, order of the calculation, etc.) that affect the kinematics of the decay products of the Higgs boson and the jets produced in association with it. Thus measurements in the fiducial region ensure that the experimental measurements factorize from the large uncertainties that come from this model dependence. Residual model dependence coming from signal modelling, signal efficiencies and migrations between bins is evaluated and found to have a negligible impact.

The extraction of the signal yield is performed with a likelihood fit to the invariant mass of selected diphotons as shown in Figure 2 for the case of the total fiducial region. This extraction comes with sizable systematic uncertainty from the background modelling and photon energy resolution affecting the signal modelling. The signal yield is also extracted in bins of the observable 
under study in order to extract differential cross section measurements. In all cases, the extracted signal yields are corrected to particle-level with correction factors obtained from simulation. The systematic uncertainties of the correction factors are generally smaller than those affecting the signal yield extraction and come from photon efficiencies, but become larger in the case of bins of jet-related observables due to jet energy scale and resolution uncertainties.

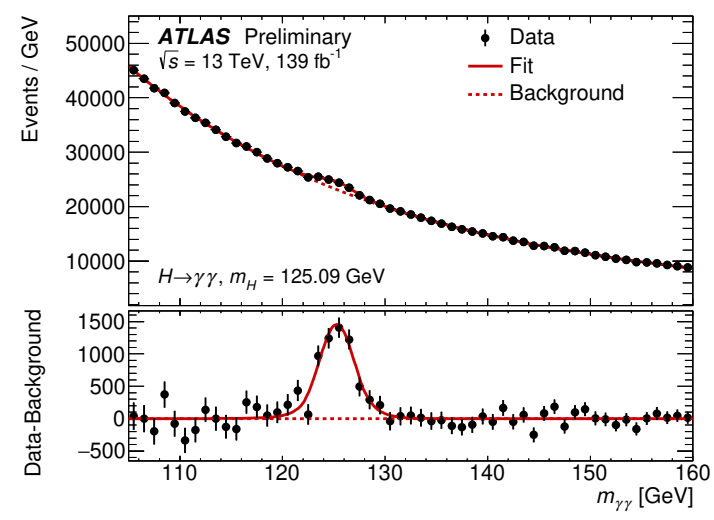

Figure 2: Diphoton invariant mass distribution in the data, shown with the fit used to extract the signal yield in the total fiducial region [6].

The total fiducial cross section times branching ratio for $H \rightarrow \gamma \gamma$ production is measured to be $\sigma_{\text {fid }}=65.2 \pm 4.5$ (stat.) \pm 5.6 (syst.) \pm 0.3 (theo.) fb, that is in good agreement with the SM prediction of $63.6 \pm 3.3 \mathrm{fb}$ [2]. Differential cross sections are shown in Figure 3 as a function of the diphoton transverse momentum, $p_{\mathrm{T}}^{\gamma \gamma}$, the leading jet transverse momentum, $p_{\mathrm{T}}^{j_{1}}$, and the azimuthal angular difference between the two leading jets, $\Delta \phi_{j j}$ (ordering jets according to their transverse momentum). Also measured, though not shown here, is the rapidity of the diphoton system, the jet multiplicity and the invariant mass of the two leading jets. All differential measurements have a high level of compatibility with the SM predictions with $p$-values above $56 \%$ in all cases.

\section{EFT interpretations and limits on charm Yukawa coupling modication}

The measured $H \rightarrow \gamma \gamma$ fiducial cross sections are used to probe the strength and tensor structure of the Higgs-boson interactions with SM particles in the context of an Effective Field Theory (EFT) [6]. From various available options, the Higgs Effective Lagrangian and SMEFT extensions of the SM Lagrangian that introduce dimension-6 operators for CP-even and CP-odd interactions are used in their SILH and Warsaw bases, respectively. The strength of each interaction is expressed by the value of its accompanying Wilson coefficient.

The focus of this analysis is on Wilson coefficients of operators that affect mostly the ggF, vector-boson fusion (VBF) and $V H$ production modes to which the measured distributions $\left(p_{\mathrm{T}}^{\gamma \gamma}\right.$, $N_{\text {jets }}, p_{\mathrm{T}}^{j_{1}}, m_{j j}$ and $\left.\Delta \phi_{j j}\right)$ are sensitive. The fit method is using these five one-dimensional cross sections to build a likelihood from the $\chi^{2}$ between the data and the prediction. In this approach, statistical correlations between the bins of the measurements must be taken into account, as the same events in data are populating bins in multiple observables. They enter in the likelihood in the form of a covariance matrix together with the experimental uncertainties on the data measurements 


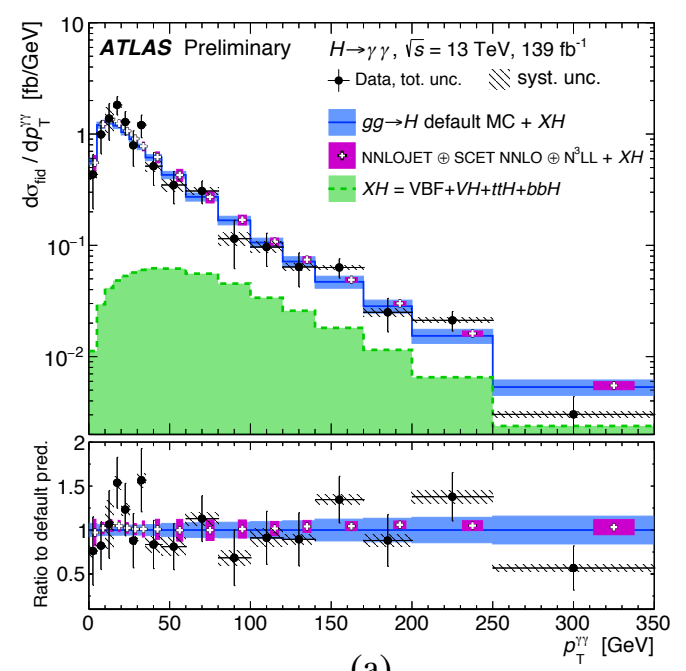

(a)

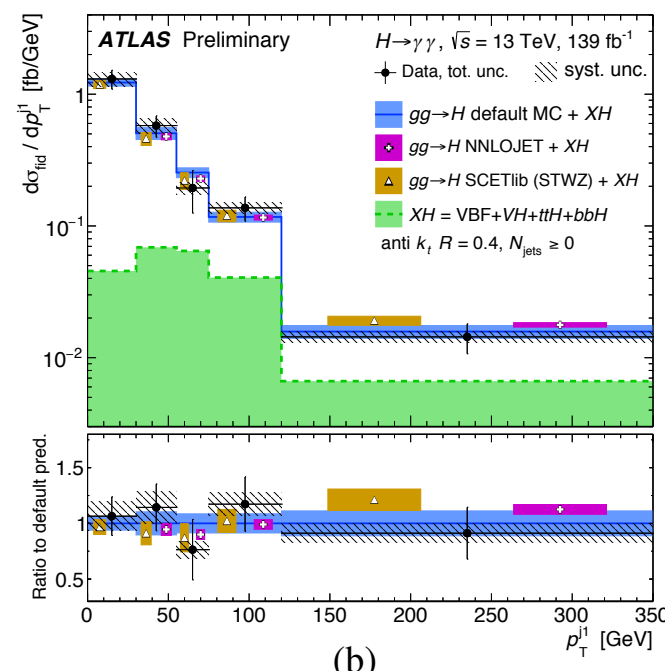

(b)

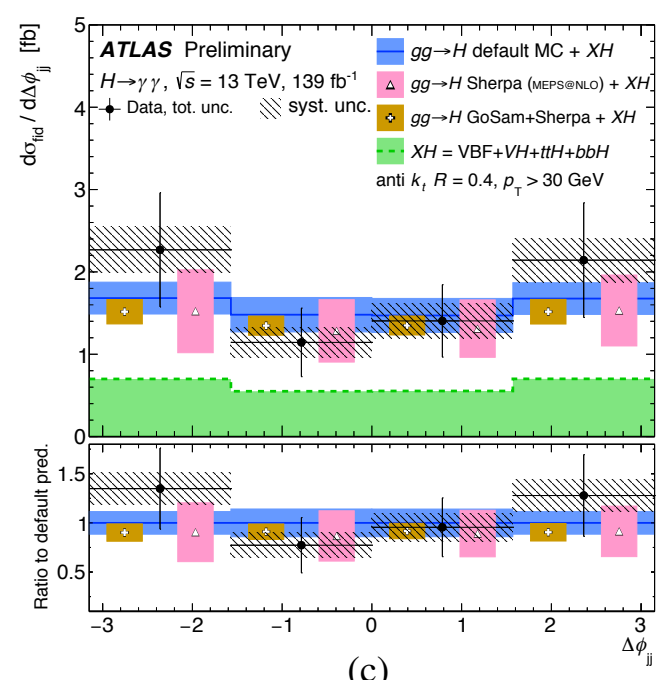

(c)

Figure 3: Measured cross sections as a function of (a) diphoton transverse momentum, (b) leadingjet transverse momentum and (c) azimuthal separation of the two leading jets, compared with multiple predictions for gluon-gluon fusion $(\mathrm{ggF})$ production added to other common Higgs boson production processes $(X H)[6]$.

and theoretical uncertainties on the predictions. The limits on the Wilson coefficients are obtained by scanning one or more coefficients at a time while setting all others to zero. As the measured cross sections are in good agreement with the SM predictions, no significant deviation from the zero is seen for any of the Wilson coefficients studied. Shown in Figure 4a are the 68\% and 95\% confidence intervals from the fits to single coefficients of the Warsaw basis of the SMEFT Lagrangian.

Another example of how the differential cross sections can be used is the interpretation in the context of light-quark Yukawa couplings of the Higgs boson. Limits are set on a potential modification of the Yukawa coupling to the charm quark compared to that predicted in the SM, $\kappa_{c}$, based on the expected effect on the shape of the inclusive $p_{\mathrm{T}}^{\gamma \gamma}$ distribution [6]. Specifically, only effects from the charm contribution to the loop in ggF production and $c \bar{c} \rightarrow H$ production are 
considered. By not accounting for the expected effect on the branching ratio and thus in the overall normalization, there is significant loss in sensitivity at the benefit of having a simpler model with fewer assumptions. The reported $95 \%$ confidence interval for $\kappa_{c}$ is $[-19,24]$ for which the expected $p_{\mathrm{T}}^{\gamma \gamma}$ shape is shown in Figure $4 \mathrm{~b}$ in comparison to the actual distribution measured in the data.

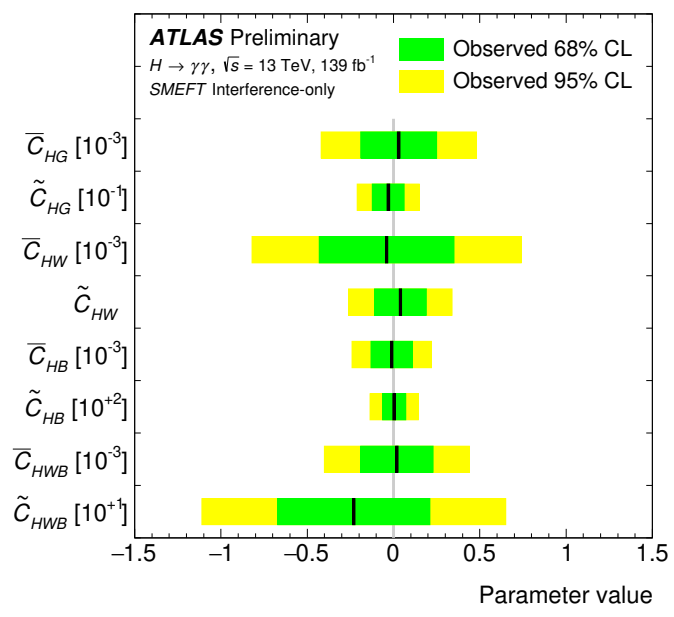

(a)

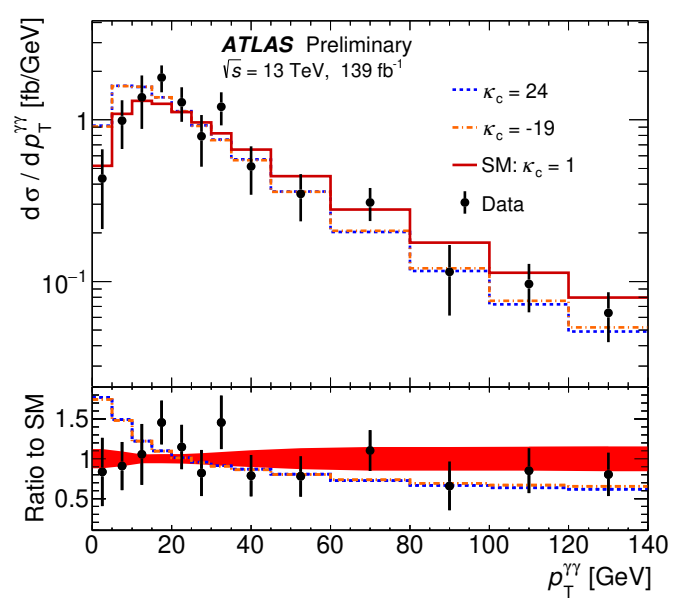

(b)

Figure 4: (a) Limits at 68\% and 95\% confidence level for Wilson coefficients of the Warsaw basis of the SMEFT Lagrangian obtained from fits to the measured $H \rightarrow \gamma \gamma$ differential cross sections. (b) Measured $p_{\mathrm{T}}^{\gamma \gamma}$ distribution in data compared to the predicted ones for $\kappa_{c}$ values corresponding to the upper and lower limits set at 95\% confidence level [6].

\section{Conclusions}

First results from the analysis of the full Run-2 dataset of $\sqrt{s}=13 \mathrm{TeV} p p$ collisions at the LHC recorded with the ATLAS detector for the $H \rightarrow \gamma \gamma$ decay were presented regarding fiducial cross sections and the single-channel observation of the $t t H$ production. Together with earlier STXS measurements, we see remarkable agreement with the SM predictions that allow to claim good understanding of the Higgs boson production and kinematics. The goal is to perform more precise tests by combining with other decay channels to reduce the statistical uncertainties. At the same time, the measurements can be used in interpretations in order to constrain new physics in the interactions of the Higgs boson with SM particles, such as the constraints presented on new CP-odd and CP-even interactions with an EFT approach, and also on possible modifications of the charm Yukawa coupling.

\section{References}

[1] ATLAS Collaboration, 2008 JINST 3 S08003.

[2] D. de Florian et al., arXiv:1610.07922 [hep-ph].

[3] N. Berger et al., arXiv:1906.02754 [hep-ph].

[4] ATLAS Collaboration, ATLAS-CONF-2018-028, http://cds.cern.ch/record/2628771.

[5] ATLAS Collaboration, ATLAS-CONF-2019-004, http://cds.cern.ch/record/2668103.

[6] ATLAS Collaboration, ATLAS-CONF-2019-029, https://cds.cern.ch/record/2682800. 